\title{
Un modèle simple d'interception du rayonnement solaire par la vigne - vérification expérimentale
}

\author{
C. Riou, C. Valancogne et P. Pieri \\ INRA, Laboratoire de Bioclimatologie, Domaine de la Grande Ferrade, F-33140 Pont-de-la-Maye, France
}

(reçu le 20 novembre 1987, accepté le 12 février 1989)

Résumé - La vigne traditionnelle de la région de Bordeaux, sujet de l'étude, présente des caractéristiques de forme bien définies et un indice foliaire faible.

Ce travail utilise une représentation géométrique simple de la vigne et de sa porosité; il permet d'évaluer la part de rayonnement solaire direct et diffus absorbé par une telle vigne et réfléchi par la parcelle. Les mesures effectuées fournissent une bonne vérification du modèle qui permet ensuite de simuler l'énergie absorbée par des vignes d'orientation différente à plusieurs périodes de l'année.

viticulture - énergie solaire - modélisation - orientation - culture en rangs

Summary - A simple model for interception of solar radiation by a vineyard - comparison with field data. The traditional vineyard near Bordeaux has simple forms and low leaf area density.

This study uses a simple geometric model for vine and its porosity. It allows an estimation of the absorption of direct and diffuse solar energy and of the vineyard. Experimental field data are in a good agreement with the model. $A$ simulation of absorbed solar energy reflection by vineyards with two row orientations at different dates is proposed.

viticulture - solar energy - modelling - row - orientation - row-crop

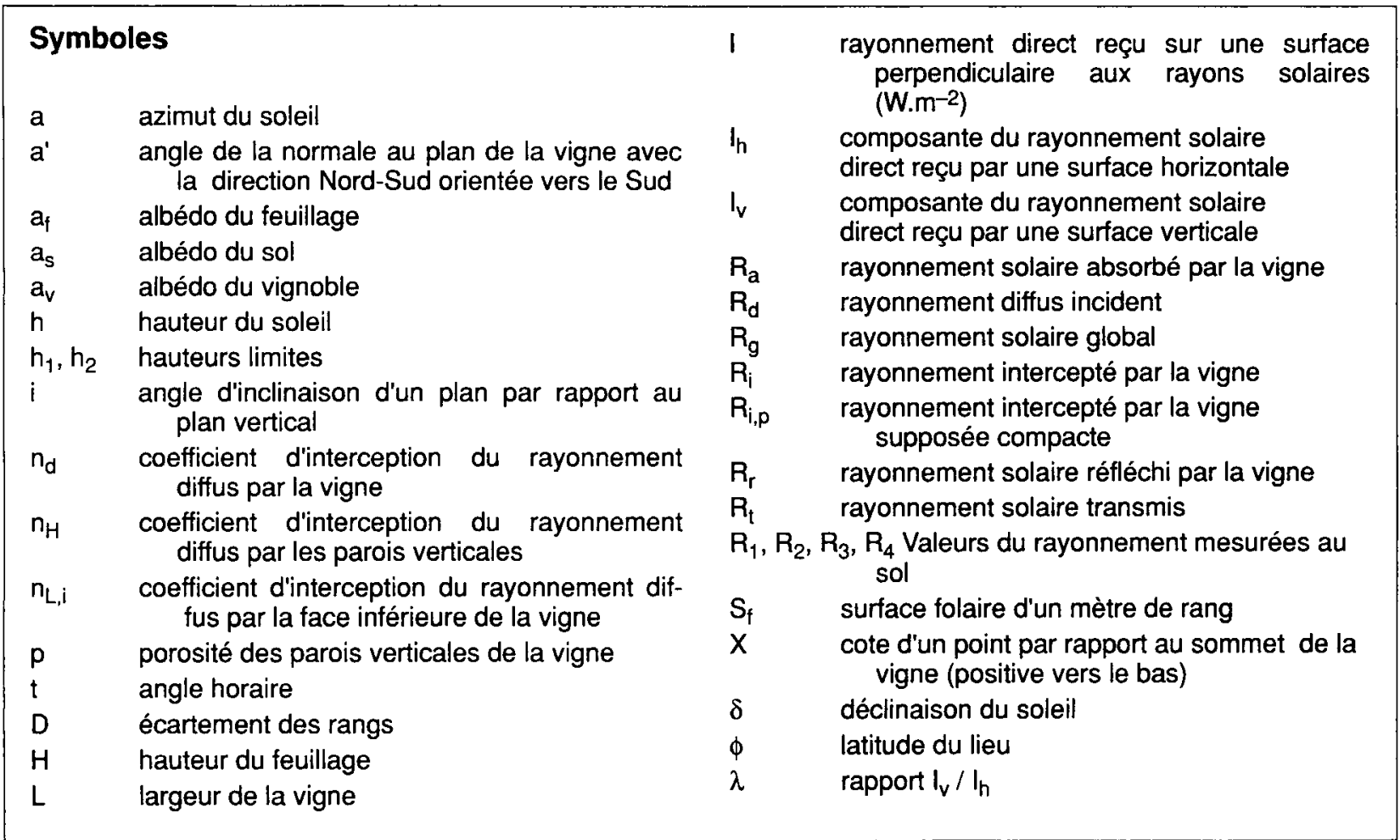




\section{Introduction}

Ce travail se donne pour objet de modéliser l'interception des rayonnements solaires direct et diffus par une vigne traditionnelle du vignoble de la région de Bordeaux. Les modèles proposés dans la littérature scientifique ne sont pas en général adaptés au problème posé, parce qu'ils limitent leur portée en ne prenant pas en compte la porosité de la végétation (Jackson et Palmer 1971; Smart 1973) ou qu'ils concernent des formes complexes assimilées à des ellipsoïdes, à indice foliaire élevé, ce qui introduit une extinction du rayonnement de type exponentiel (Charles-Edwards et Thorpe 1976; Mann et al., 1980; Norman et Welles 1983). II y a cependant deux études qui se rapprochent de la nôtre par le modèle géométrique de la culture : le modèle de Allen (1974), mais il concerne uniquement le rayonnement direct et utilise également la loi de Bouguer, et les travaux de Fuchs et Stanhill (1980) qui étudient l'interception du rayonnement direct par le sorgho et s'appuient sur des photographies hémisphériques.

Ajoutons que certains des modèles cités concernent uniquement le rayonnement photosynthétique actif, alors que notre but ici est d'obtenir des éléments pour une étude complète du bilan énergétique de la vigne; en conséquence, on adoptera ici pour la feuille un coefficient de réflexion qui est une valeur moyenne établie pour l'ensemble des radiations du spectre solaire.

La vigne sur laquelle le modèle a été vérifié et qui est très répandue dans la région de Bordeaux présente en effet quelques particularités qui rendent inadéquats la plupart des modèles existants :

- son enveloppe a une forme simple imposée par la taille, proche de celle d'un parallélépipède;

- elle a un indice foliaire faible, et on ne peut lui appliquer la loi de Bouguer, qui suppose des réflexions multiples à l'intérieur du feuillage;

- elle a une porosité notable, surtout dans ses parois verticales.

Dans cet article, on se propose de partir de ces données et de présenter un modèle simple, permettant d'évaluer l'interception par la vigne du rayonnement solaire direct et celle des rayonnements solaires diffusés par le ciel et par le sol; le rayonnement réfléchi sera également modélisé et les évaluations seront comparées à des mesures effectuées pendant une petite période au voisinage de l'équinoxe; les résultats seront considérés comme une validation du modèle proposé. Pour terminer, l'énergie solaire absorbée sera calculée par simulation, pour différentes époques de l'année et deux orientations des rangs.

\section{Modélisation}

\section{Forme et porosité de la vigne}

Le feuillage, maintenu par la taille dans des limites strictes, est inscrit dans un volume en forme de parallélépipède; ce volume présente des vides, mais ceux-ci sont surtout notables dans les parois verticales, alors que la vigne vue de dessus ou de dessous est beaucoup plus compacte. La porosité est surtout faite de grandes plages vides qui correspondent à des zones où plusieurs feuilles manquent. Le modèle géométrique adopté est une enveloppe parallélépipédique limitée par deux surfaces horizontales pleines de largeur $L$ et deux parois verticales de hauteur $\mathrm{H}$ et de porosité totale $\mathrm{p}$ qu'on supposera indépendante de la direction des rayons lumineux; $p$ est défini comme la fraction de la surface verticale ne donnant pas lieu à de l'interception. Deux modèles physiques sont en accord avec cette hypothèse; dans le premier, chaque face est percée de multiples vides répartis de façon aléatoire avec une porosité $\sqrt{p}$, l'ensemble des deux faces ayant une porosité $\mathrm{p}$. L'autre modèle, plus proche de la réalité, correspond à de grandes ouvertures à bords minces, où l'épaisseur de la vigne n'intervient pas sur la variation de la porosité en fonction de l'angle d'incidence du rayon lumineux. Ces ouvertures sont d'ailleurs localisées dans la partie supérieure de la vigne et dans une moindre mesure dans la partie inférieure.

Grâce à des photographies numérisées, il a été possible de mesurer la porosité $p$ à laquelle on a donné la valeur d'1/3. On peut noter que si les faces du volume considéré étaient tapissées de feuilles horizontales ou verticales suivant le cas, la surface foliaire pour $1 \mathrm{~m}$ de rang serait en $\mathrm{m}^{2}$ :

$$
S_{f}=2 L+2(1-p) H
$$

soit dans le cas de la vigne étudiée :

$$
\begin{aligned}
& L=0,3 \quad H=0,9 \quad p=1 / 3 \\
& S_{f}=1,8 \mathrm{~m}^{2}
\end{aligned}
$$

Cette valeur correspond exactement à la surface de sol comprise entre deux rangs et ce taux de surface foliaire de $10000 \mathrm{~m}^{2}$ par hectare est très voisin de celui qui a été mesuré sur une vigne traditionnelle semblable à celle qui nous a servi de modèle, soit $10500 \mathrm{~m}^{2} / \mathrm{ha}$ (Carbonneau et Huglin, 1980). En fait, les feuilles sont ici légèrement obliques. 
Interception du rayonnement direct par un parallélépipède supposé plein

\section{Cas des parois verticales}

Le problème est traité en deux dimensions, la rangée de vigne étant supposée infinie. Soit a l'azimut du soleil (angle formé avec la direction Sud), a', l'angle de la normale au plan de la vigne avec la direction Nord-Sud orientée vers le Sud, et I le rayonnement direct, la composante de ce dernier qui intéresse les parois verticales de la vigne est alors (Fig. 1):

$$
I_{v}=I \cos h \cos \left(a-a^{\prime}\right)
$$

et le rapport avec la composante verticale $I_{h}$ :

$$
\lambda=\frac{I_{v}}{I_{h}}=\frac{\cos h \cos \left(a-a^{\prime}\right)}{\sinh }
$$

Par ailleurs, d'après les formules de l'astronomie:

$$
\cos a=\frac{\sin \phi \sin h-\sin \delta}{\cos \phi \cos h}
$$

et

$$
\sin a=\frac{\cos \delta \sin t}{\cos h}
$$

D'où :

$$
\lambda=\cos a^{\prime} \operatorname{tg} \phi-\frac{\sin \delta \cos a^{\prime}}{\sin h \cos \phi}+\frac{\sin a^{\prime} \cos \delta \sin t}{\sinh }(5)
$$

Deux cas sont intéressants parce qu'ils sont fréquents, (1) celui des vignes orientées NordSud (les plus nombreuses), et (2) celui des vignes orientées Est-Ouest.

$$
\begin{aligned}
& \text { On a pour (1) } a^{\prime}=(\Pi / 2) \text { et } \\
& \lambda_{1}=\left|\frac{\cos \delta \sin t}{\sin h}\right| \\
& \text { et pour (2) } a^{\prime}=0 \text { et : } \\
& \lambda_{2}=\left|\operatorname{tg} \phi-\frac{\sin \delta}{\sin h \cos \phi}\right|
\end{aligned}
$$

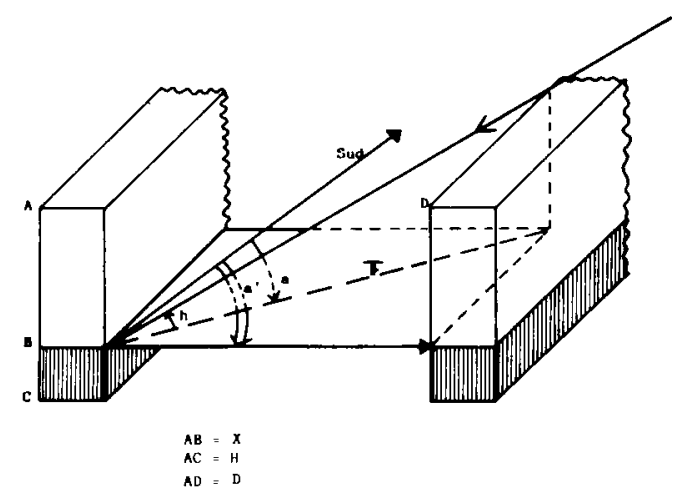

Fig. 1. Géométrie de l'ombre portée par un rang sur un rang voisin.

\section{Expression en l'absence d'ombre portée}

Le soleil n'atteignant qu'un côté $\mathrm{H}$ du parallélépipède, l'énergie interceptée directement sera $\lambda \mathrm{HI}_{h}$, à laquelle il faut ajouter ce que reçoit la surface horizontale de la vigne $L_{h}$. Si l'on ramène cette énergie à l'unité de surface totale de sol, on aboutit (en l'absence d'ombre portée et dans l'hypothèse d'un volume plein) à l'expression :

$$
R_{i, p}=\left(I_{h} / D\right)(\lambda H+L)
$$

\section{Ombre portée}

En dessous d'une certaine hauteur du soleil, un rang crée une zone d'ombre sur le rang voisin et la hauteur éclairée de chaque rang (supposé infiniment long) est alors (voir Fig. 1):

$$
X=T \operatorname{tgh}
$$

avec

$$
T=\frac{D-L}{\cos \left(a-a^{\prime}\right)}
$$

soit

$$
X=\frac{(D-L) \operatorname{tgh}}{\cos \left(a-a^{\prime}\right)}=\frac{D-L}{\lambda}
$$

On en déduit que la vigne est complètement éclairée quand $\mathrm{X}=\mathrm{H}$ hauteur du feuillage, soit quand

$$
\lambda=\lambda_{0}=\frac{D-L}{H}
$$

Avant que cette phase soit atteinte $\left(\lambda>\lambda_{0}\right)$, la fraction de rayonnement direct reçue par la paroi verticale de la vigne prend la valeur:

$$
\frac{X I_{v}}{D}=\frac{X}{D} \lambda I_{h}=\frac{D-L}{D} I_{h}
$$

A ceci il faut ajouter la part reçue par la surface horizontale $\left(I_{h} L / D\right)$ et la vigne reçoit alors un rayonnement $I_{h}$, identique au rayonnement reçu par une surface horizontale continue, ce qui traduit simplement le fait que le sol est totalement à l'ombre.

\section{Interception du rayonnement diffus}

\section{Cas des parois verticales (supposées pleines)}

$\mathrm{Si}$ l'on considère une mince bande de vigne, située à une même distance $X$ du sommet vigne, elle ne reçoit du ciel qu'une portion du rayonnement diffus qu'on peut calculer (dans l'hypothèse d'une luminance uniforme du ciel) par la formule donnant l'éclairement fourni par un élément de ciel de hauteur $h$ et d'azimut a sur un plan vertical.

$$
\frac{1}{\Pi} \int_{0}^{\pi} \int_{h(a)}^{\frac{\pi}{2}} \cos \left(a-a^{\prime}\right) \cos ^{2} h \cdot d a \cdot d h
$$


h (a) est ici la hauteur limite due à la proximité du rang parallèle qu'on peut calculer par :

$$
\operatorname{tg} h(a)=\frac{X}{D-L} \cos \left(a-a^{\prime}\right)
$$

L'intégrale double a pour valeur :

$$
\sin ^{2}\left(\frac{i}{2}\right)
$$

où i est $\operatorname{Arctg}\left(\frac{D-L}{X}\right)$

C'est l'angle avec le plan vertical du plan passant par la droite située à une distance $X$ du sommet de la vigne et l'arête la plus proche du sommet du rang voisin.

L'intégration le long de la paroi verticale, en exprimant la variable $X$ en fonction de $i$, conduit à :

$(D-L) \int_{i_{0}}^{\frac{\pi}{2}} \frac{\sin ^{2}\left(\frac{i}{2}\right) d i}{\sin ^{2} i}$
$i_{0}=\operatorname{Arctg} \frac{D-L}{H}$

On obtient finalement:

$$
\frac{D-L}{2}\left(1-\operatorname{tg} \frac{\mathrm{i}_{0}}{2}\right)
$$

Soit pour l'ensemble des deux parois et par unité de surface :

$$
\mathrm{n}_{\mathrm{H}}=\frac{\mathrm{D}-\mathrm{L}}{\mathrm{D}}\left(1-\operatorname{tg} \frac{\mathrm{i}_{\circ}}{2}\right)
$$

\section{Cas des parois horizontales}

Si la partie supérieure de la vigne reçoit la totalité du rayonnement diffus, la partie horizontale inférieure n'en reçoit qu'une fraction limitée par les deux rangs de vigne voisins (et par les parois verticales poreuses, qu'on supposera d'abord inexistantes). On admettra que tout point de la surface reçoit la même énergie que les points de l'axe central, ce qui est justifié par la faible largeur de la vigne par rapport à l'écartement des rangs; on a alors :

$$
n_{L, i}=\frac{2 L}{D} \int_{0}^{\pi} \int_{h_{1}}^{h_{2}} \cos h \cdot \sin h d a \cdot d h
$$

$h_{1}$ et $h_{2}$ sont les hauteurs limites imposées par la partie supérieure et la rangée de vigne voisine (Fig. 2a), avec :

$$
\begin{aligned}
& \operatorname{tgh}_{1}=\frac{2 H}{L} \sin a \\
& \operatorname{tgh}_{2}=\frac{H}{D-L / 2} \sin a
\end{aligned}
$$

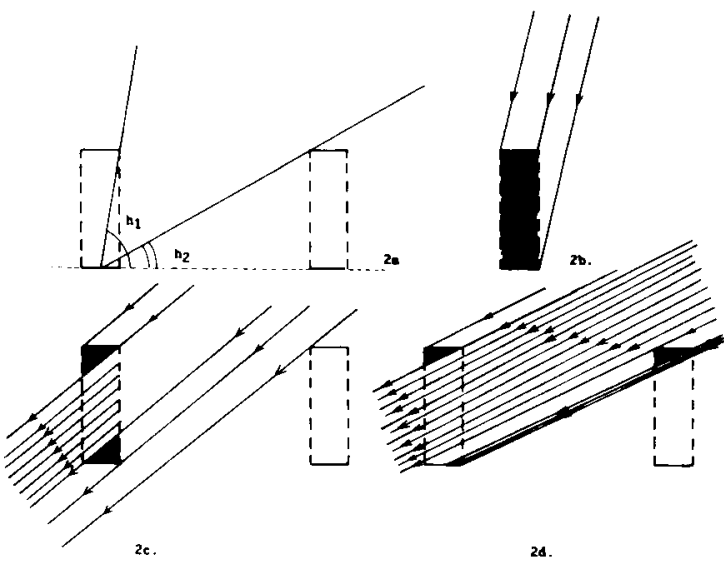

Fig. 2. 2a. Angles utilisés pour le calcul du rayonnement diffus intercepté par la surface horizontale inférieure de la vigne. 2b. Interception totale du rayonnement direct par la vigne $(\lambda<$ $(\mathrm{L} / \mathrm{H})$ ).

2c. Interception du rayonnement direct dans le cas $(L / H)<$ $\lambda<(\mathrm{D}-\mathrm{L} / \mathrm{H})$.

2d. Interception du rayonnement direct dans le cas $(D / H)<\lambda$ $<(\mathrm{D}+\mathrm{L} / \mathrm{H})$.

Les hachures serrées correspondent à une interception totale; les hachures espacées correspondent à une interception partielle due à la porosité.

On montre à partir de (13) qu'on a :

$$
\mathrm{n}_{\mathrm{L}, \mathrm{i}}=\left(\frac{2 \mathrm{~L}}{\mathrm{D}}\right)\left[\sin ^{2}\left(\frac{\mathrm{h}_{1}}{2}\right)-\sin ^{2}\left(\frac{\mathrm{h}_{2}}{2}\right)\right]
$$

Interception totale du rayonnement diffusé par le ciel

Puisque seule une fraction $p$ du rayonnement diffus atteint la surface inférieure, et qu'une autre $(1-p)$ est interceptée par les parois verticales, on a finalement pour la part du rayonnement diffusé par le ciel et intercepté par la vigne, en négligeant la part du diffus transmis par les rangs de vigne voisins :

$$
n_{d}=(1-p) n_{H}+\frac{L}{D}+p n_{L, i}
$$

\section{Interception du rayonnement solaire par la vigne}

Le rayonnement solaire direct intercepté est décomposé en trois parties (Figs. 2b, 2c, 2d) :

a. Le rayonnement solaire direct reçu par la partie supérieure de la vigne, soit $L I_{h} / D$.

b. Le rayonnement direct intercepté par les parois; on applique ici au rayonnement traversant la vigne un coefficient d'interception 1-p.

c. Le rayonnement direct solaire intercepté par la partie inférieure de la vigne.

Une autre méthode de calcul consiste à retrancher du rayonnement intercepté par le parallélépipède supposé plein, la part d'énergie qui parvient au sol. On examinera successivement tous 
ment tous les cas, en partant de la position du soleil au zénith, en faisant ensuite diminuer la hauteur $h$ ou augmenter $\lambda$ et en introduisant les ombres portées (et la part d'énergie transmise) par le rang voisin, puis les rangs suivants, en se limitant au cas de trois rangées successives; la vigne absorbe en effet alors 97\% du rayonnement direct. Les relations qui suivent supposent $\mathrm{L}<(\mathrm{D} / 2)$.

$$
\text { 1. } \lambda \leq \frac{L}{H}
$$

Aucune part du rayonnement direct intercepté par la vigne ne parvient au sol (Fig. 2b).

$$
\begin{aligned}
& R_{i}=(1 / D)(\lambda H+L) I_{h}+n_{d} R_{d} \\
& \text { 2. } \frac{L}{H}<\lambda \leq \frac{D-L}{H}
\end{aligned}
$$

Il n'y a pas d'ombre portée par un rang sur le voi$\sin$.

Une partie du rayonnement direct qui traverse les deux parois verticales, est transmise au sol. Elle correspond à une fraction $p$ du rayonnement intercepté par la hauteur de vigne $H-L \lambda$, soit :

$p \lambda(H-(L / \lambda)=p(\lambda H-L)$ et le rayonnement solaire intercepté est finalement (Fig. $2 c$ ) :

$$
\begin{aligned}
& R_{i}=(1 / D)[\lambda H+L-p(\lambda H-L)] I_{h}+n_{d} R_{d} \\
& \text { 3. } \frac{D-L}{H}<\lambda \leq \frac{D}{H}
\end{aligned}
$$

Le rayonnement direct ne parvient au sol qu'à travers la vigne.

$$
\begin{aligned}
& R_{i}=(1 / D)[D-p(\lambda H-L)] I_{h}+n_{d} R_{d} \\
& \text { 4. } \frac{D}{H}<\lambda \leq \frac{D+L}{H}
\end{aligned}
$$

Une partie de l'énergie transmise par le feuillage du rang voisin est interceptée par la surface inférieure de la vigne (Fig. $2 \mathrm{~d}$ ).

$$
\begin{aligned}
& R_{i}=(1 / D)[D-p(2 D-L-\lambda H)] I_{h}+n_{d} R_{d} \\
& \text { 5. } \frac{D+L}{H}<\lambda \leq \frac{2 D-L}{H}
\end{aligned}
$$

Le deuxième rang des vignes voisines est pris en compte et le calcul conduit à :

$$
\begin{aligned}
R_{i}=(1 / D)[D-p & (2 D-L-\lambda H)- \\
& \left.p^{2}(\lambda H-D-L)\right] I_{h}+n_{d} R_{d}
\end{aligned}
$$

Le calcul est effectué ainsi jusqu'à : $\lambda \leq(3 D / H)$

$$
R_{i}=(1 / D)\left[D-p^{3}(D-L)\right] I_{h}+n_{d} R_{d}
$$

Interception du rayonnement réfléchi par le sol et expression finale des rayonnements réfléchis et absorbés par la vigne

Le même raisonnement qui a conduit au calcul de $n_{d}$ pour le rayonnement diffusé par le ciel s'applique au calcul de l'interception par la vigne du rayonnement réfléchi par le sol.

On supposera d'abord que le rayonnement solaire (direct et diffus) intercepté par la vigne $R_{i}$ est réfléchi uniquement vers le haut en s'appuyant sur le fait que les feuilles ne sont pas dans un plan vertical mais légèrement inclinées (60 à $70^{\circ}$ environ avec un plan horizontal); o $n$ négligera donc l'énergie renvoyée par réflexion vers le rang voisin; en revanche, pour le rayonnement réfléchi par la vigne et venant du sol, on admettra qu'il se répartit uniformément vers le haut et vers le bas. Enfin, on négligera toutes les autres réflexions.

Soit $a_{f}$ l'albédo du feuillage (valeur moyenne pour l'ensemble du spectre), $a_{s}$ celui du sol, $R_{a}$ et $R_{r}$ les énergies absorbées et réfléchies par la vigne, on a :

$$
\begin{aligned}
& R_{a}=\left(1-a_{f}\right) R_{j}+a_{s} n_{d}\left(1-a_{f}\right)\left(R_{g}-R_{i}\right) \\
& \text { ou } \\
& R_{a}=\left(1-a_{f}\right)\left[\left(1-a_{s} n_{d}\right) R_{i}+a_{s} n_{d} R_{g}\right] \\
& R_{r}=a_{f} R_{i}+\left(1-n_{d}\right) a_{s}\left(R_{g}-R_{i}\right)+ \\
& 0,5 n_{d} a_{s}\left(R_{g}-R_{i}\right) a_{f}
\end{aligned}
$$

et finalement

$$
\begin{array}{r}
R_{r}=\left[a_{f}-\left(1-n_{d}\right) a_{s}-0,5 n_{d} a_{s} a_{f}\right] R_{i}+ \\
{\left[\left(1-n_{d}\right) a_{s}+0,5 n_{d} a_{s} a_{f}\right] R_{g}}
\end{array}
$$

\section{Vérification du modèle}

\section{Dispositif expérimental}

Le site choisi pour les mesures est implanté dans le domaine de Latresne. Des mesures de vitesse de vent ont été effectuées sur l'emplacement adopté qui a été décrit à cette occasion (Riou et al., 1987).

La vigne est orientée Nord-Sud; l'écartement des rangs est d'1,8 m, la hauteur totale au-dessus du sol de $1,5 \mathrm{~m}$, avec un feuillage de hauteur $H$ de $0,9 \mathrm{~m}$, la largeur $L$ de la vigne est de $0,3 \mathrm{~m}$ et l'écartement des pieds sur un rang de 1,1 $\mathrm{m}$.

Quatre pyranomètres placés près du sol sont répartis entre deux rangs sur un axe Ouest-Est, aux emplacements suivants : sur le rang, 1/4 d'intervalle à l'Ouest, moitié de l'intervalle, 3/4 d'intervalle à l'Ouest. Les rayonnements incidents $R_{g}$ et réfléchis sont mesurés en permanence à $3,5 \mathrm{~m}$ au-dessus du sol ainsi que le rayonnement diffus $R_{d}$, ce qui permet d'obtenir $I_{h}=R_{g}$ - $R_{d}$. Les valeurs fournies par les appareils sont enregistrées sur une centrale de mesure et les moyennes calculées toutes les 15 minutes. La période des mesures va du 25 au 30 septembre 
1986, période pendant laquelle il y eut cinq belles journées consécutives.

L'albédo du sol est mesuré sur une surface identique à la zone intercalaire des vignes, près du sol à l'extrémité de cette zone, hors de l'influence du feuillage.

\section{Résultats des mesures}

Le rayonnement absorbé par la vigne est calculé de la façon suivante :

$$
\begin{aligned}
& R_{a}=R_{g}-a_{v} R_{g}+a_{s} R_{t}-R_{t} \\
& R_{t}=1 / 4\left(R_{1}+R_{2}+R_{3}+R_{4}\right)
\end{aligned}
$$

$a_{v} R_{g}$ est le rayonnement réfléchi mesuré audessus du vignoble et $a_{v}$ l'albédo de la parcelle qui varie suivant les contributions relatives du sol et de la vigne et, donc, suivant la hauteur du soleil.

$R_{1}, R_{2}, R_{3}, R_{4}$ sont les rayonnements mesurés au sol.

Les résultats sont indiqués dans le Tableau I.

Dans l'ensemble, les valeurs moyennes journalières de l'albédo de la parcelle et du coefficient d'absorption de la vigne sont peu variables, si l'on omet la journée de très faible ensoleillement. La moyenne des coefficients journaliers de réflexion des jours à moins de $30 \%$ de diffus est de $15,3(15,1$ à 15,8). La valeur moyenne du coefficient d'absorption est pour ces mêmes journées de 41,0 , avec des valeurs allant de 39,3 à 42,7 .

Au cours de la journée, l'albédo de la parcelle, qui est de $21 \%$ au lever du soleil, diminue rapidement jusqu'à $14 \%$ vers midi, puis réaugmente d'abord lentement, puis rapidement vers le coucher du soleil (19 à 20\%). Le coefficient d'absorption qui est voisin de $50 \%$ au lever du soleil diminue aux environs de $25 \%$ vers midi puis remonte au voisinage de $60 \%$ vers le soir.
Comparaison des valeurs mesurées et calculées par le modèle

Le Tableau I établit la comparaison des valeurs moyennes journalières mesurées et calculées à partir du modèle pour les rayonnements solaires absorbés et réfléchis, tandis que sur les Figures 3,4 et 5 la comparaison est effectuée pour les valeurs moyennes établies pour un pas de temps de 15 minutes; la valeur adoptée pour l'albédo du feuillage est 0,20 (moyenne des valeurs du coefficient de réflexion différent suivant la longueur d'onde, et plus élevé dans le proche infrarouge $-40 \%$ - que dans le visible $-5 \%$ ); l'albédo mesuré du sol sec est 0,18 .

La comparaison est satisfaisante, même pour les petits pas de temps; la dispersion est plus forte pour les valeurs élevées et dans le cas du

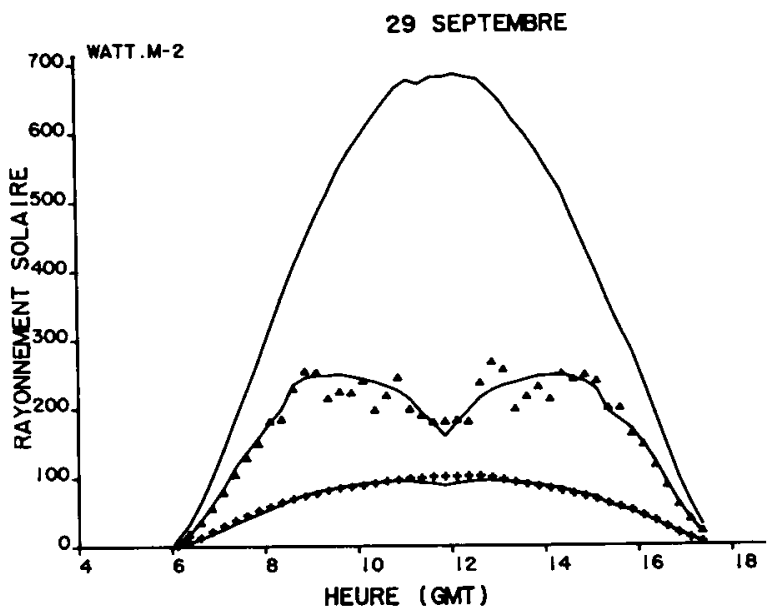

Fig. 3. Valeurs mesurées et calculées des énergies solaires absorbées par la vigne et réfléchies par le vignoble en fonction du temps. - ligne supérieure : Rayonnement global. L ligne moyenne : Rayonnement absorbé par la vigne calculé par le modèle. __ ligne inférieure : Rayonnement réfléchi par le vignoble calculé par le modèle. $\mathbf{A}$ : Rayonnement absorbé par la vigne; point expérimental. + : Rayonnement réfléchi par le vignoble; point expérimental.

Tableau I. Valeurs journalières calculées et mesurées des énergies solaires absorbées par la vigne et réfléchies par le vignoble pour 6 jours consécutifs aux environs de l'équinoxe d'automne (vigne traditionnelle, 25 au 30 septembre

\begin{tabular}{|c|c|c|c|c|c|}
\hline $\begin{array}{l}\text { Rayonnement } \\
\text { absorbé } \\
\text { mesuré } \\
\text { (W.m-2) }\end{array}$ & $\begin{array}{l}\text { Rayonnement } \\
\text { absorbé } \\
\text { calculé } \\
\left(W . m^{-2}\right)\end{array}$ & $\begin{array}{l}\text { Ecart* } \\
\text { moyen } \\
\left(W . m^{-2}\right)\end{array}$ & $\begin{array}{l}\text { Rayonnement } \\
\text { réfléchi } \\
\text { mesuré } \\
\text { (W.m-2) }\end{array}$ & $\begin{array}{l}\text { Rayonnement } \\
\text { réfléchi } \\
\text { calculé } \\
\text { (W.m-2) }\end{array}$ & $\begin{array}{l}\text { Ecart* } \\
\text { moyen } \\
\left(W \cdot m^{-2}\right)\end{array}$ \\
\hline
\end{tabular}
1987).

\begin{tabular}{|c|c|c|c|c|c|}
\hline 29 & 27 & 3 & 8 & 10 & 2 \\
\hline 153 & 158 & 22 & 59 & 59 & 4 \\
\hline 156 & 159 & 20 & 59 & 58 & 3 \\
\hline 168 & 170 & 18 & 61 & 61 & 4 \\
\hline 172 & 177 & 17 & 65 & 63 & 4 \\
\hline 180 & 180 & 21 & 65 & 64 & 5 \\
\hline
\end{tabular}

"Ecart moyen : somme du carré des écarts entre valeurs mesurées et calculées sur 15 min divisée par le nombre d'observations. 


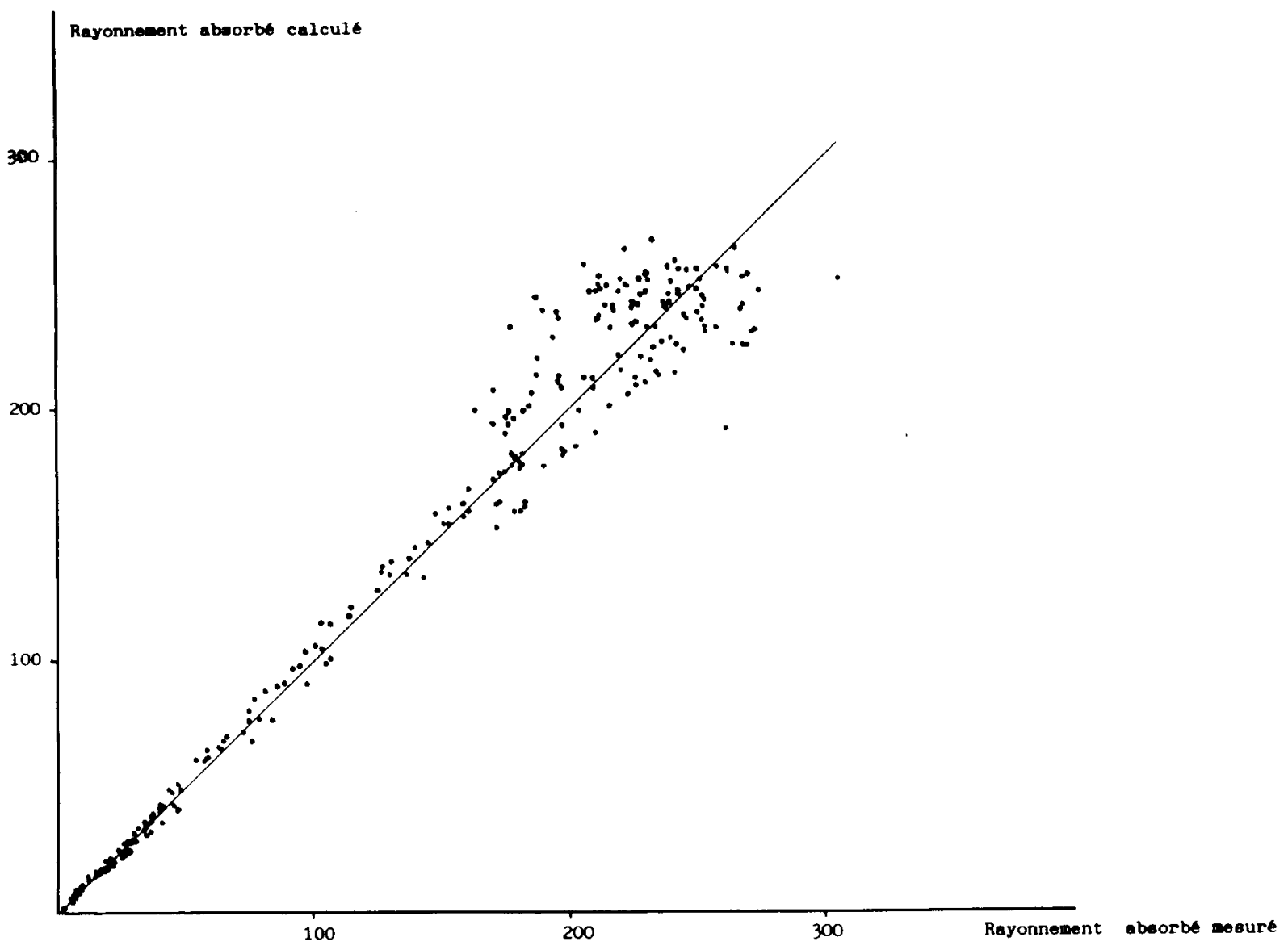

Fig. 4. Rayonnement absorbé par la vigne calculé et mesuré. Chaque point représente la moyenne de $15 \mathrm{~min}$.

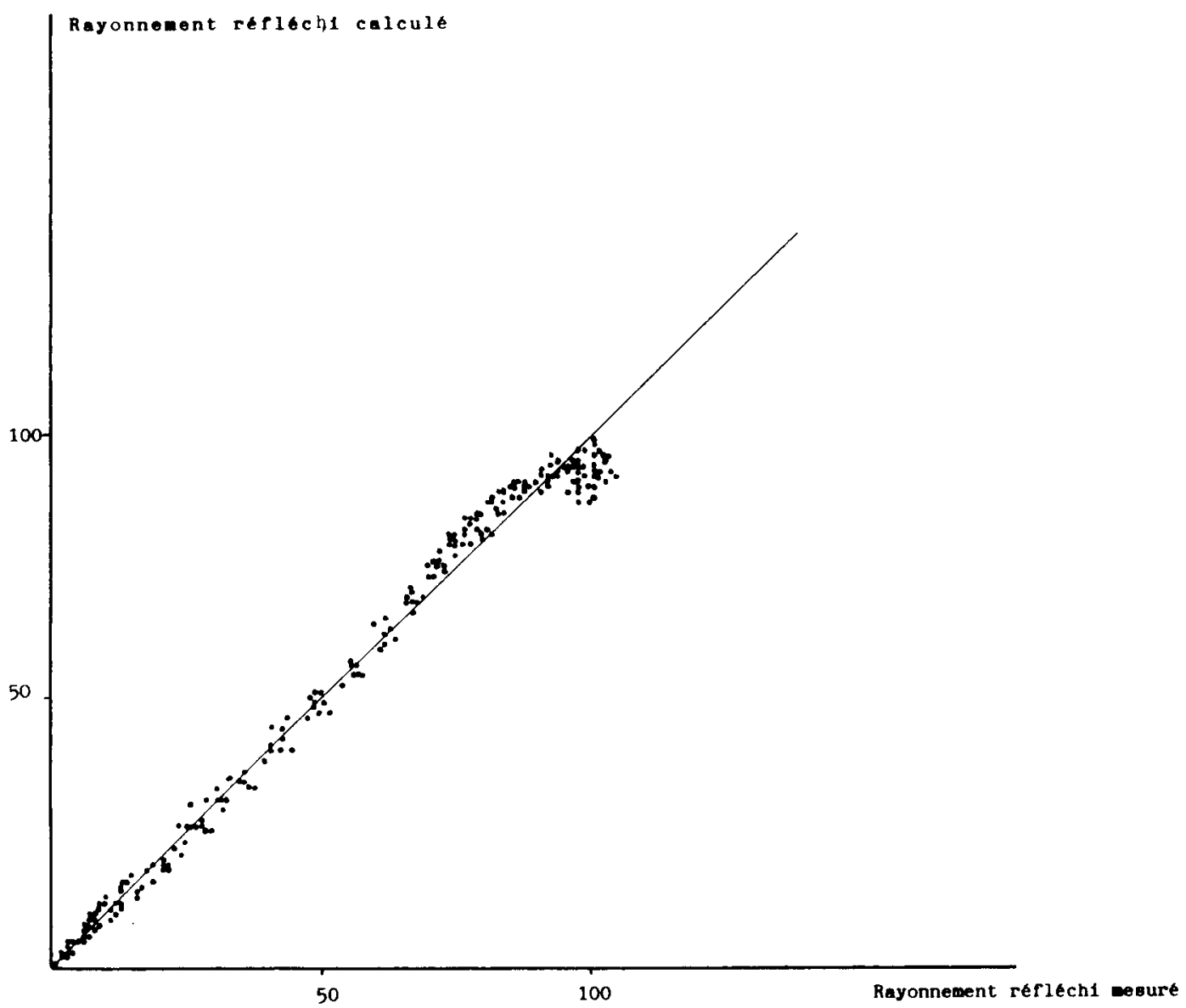

Fig. 5. Rayonnement réfléchi par la parcelle de vigne, calculé et mesuré. 
rayonnement réfléchi, il y a une légère sous-évaluation par le modèle aux environs de midi (moins de 10\%), sans qu'il soit possible d'interpréter ce résultat. Rappelons que l'irrégularité du feuillage, le nombre réduit de pyranomètres, la position de l'albédomètre sont autant d'éléments qui peuvent influencer la comparaison effectuée; celle-ci confirme cependant l'intérêt du modèle adopté.

\section{Simulation des rayonnements absorbés par la vigne selon l'orientation des rangs}

Utilisant le modèle précédent, cette simulation est faite pour deux orientations de la vigne traditionnelle, Nord-Sud et Est-Ouest (ces deux orien- tations sont présentes dans la région de Bordeaux avec une préférence marquée pour l'orientation Nord-Sud), à trois moments de l'année : près du solstice d'été, le 20 août qui correspond à la période de véraison et l'équinoxe. Les valeurs des rayonnements global et diffus sont des valeurs réelles enregistrées lors de journées de ciel clair.

Le Tableau II et les Figures 6, 7 et 8 montrent qu'au solstice, l'orientation Nord-Sud entraîne une augmentation du taux d'énergie solaire absorbée par rapport à l'orientation Est-Ouest $(+27 \%)$ alors que c'est l'inverse au moment de l'équinoxe $(-8 \%)$; dans ce dernier cas les différences sont cependant assez faibles. Le 20 août, c'est encore l'orientation N-S qui bénéficie de l'ensoleillement le plus grand $(+15 \%)$.

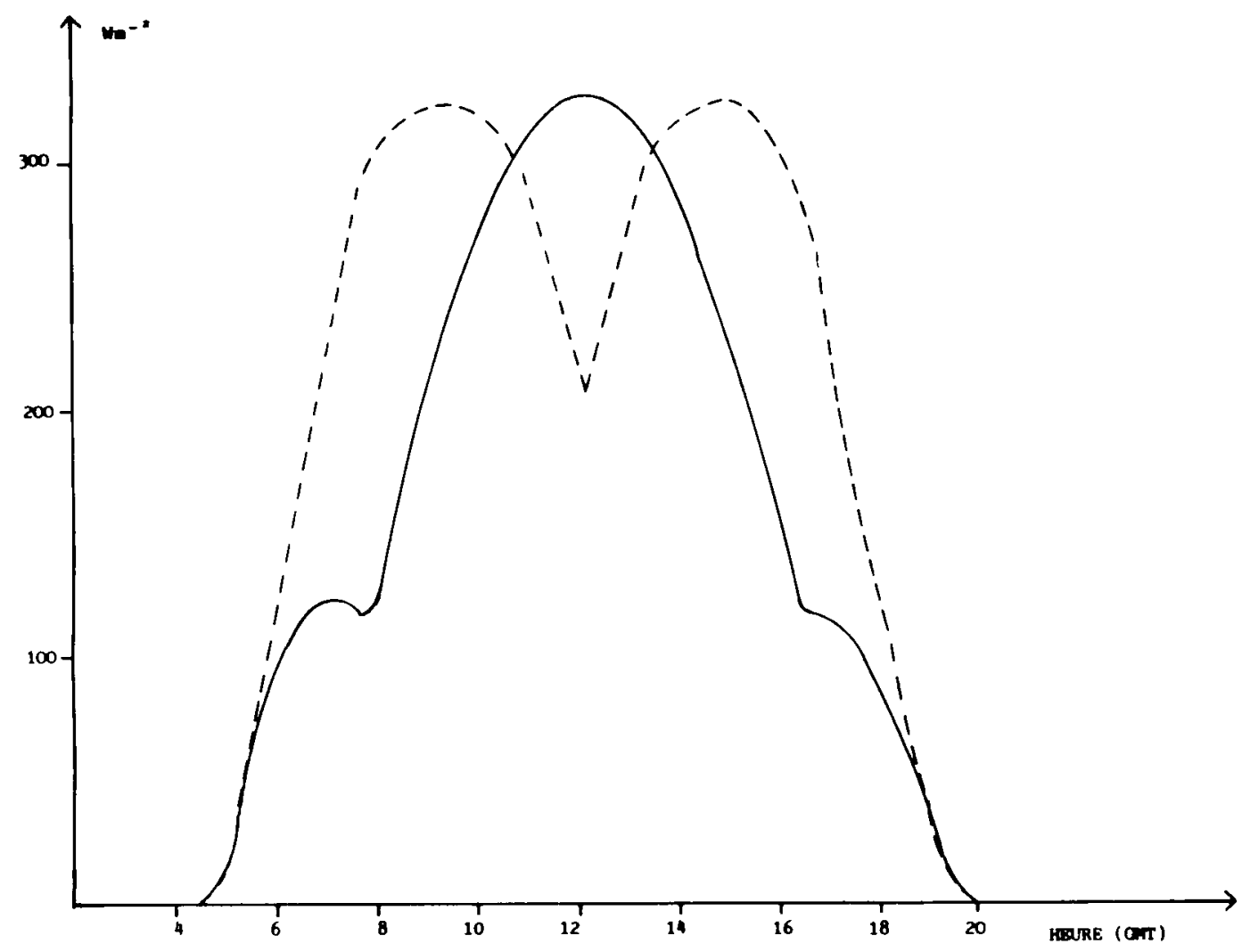

Fig. 6 à 8. Rayonnement solaire absorbé par la vigne obtenu par le modèle. ___ : orientation des rangs de vigne Est-Ouest. - - - : orientation des rangs de vigne Nord-Sud.

Fig. 6. Solstice d'été

Tableau II. Comparaison des énergies journalières absorbées par la vigne pour deux orientations différentes et trois périodes de l'année. $\mathrm{Rg}$, Ra en $10^{3} \mathrm{Jm}^{-2}$ jour-1, $\mathrm{Ra} / \mathrm{Rg}$ en \%.

\begin{tabular}{|c|c|c|c|c|c|c|c|c|c|}
\hline \multirow[t]{2}{*}{ Orientation } & \multicolumn{3}{|c|}{29 juin } & \multicolumn{3}{|c|}{20 août } & \multicolumn{3}{|c|}{28 septembre } \\
\hline & $R g$ & $R a$ & $R a / R g$ & $R g$ & $R a$ & $R a / R g$ & $R g$ & $R a$ & $R a / R g$ \\
\hline N.S. & 30.803 & 12.362 & 40,1 & 24.603 & 10.244 & 41,6 & 17.047 & 7.178 & 42,1 \\
\hline E.O. & 30.803 & 9.697 & 31,5 & 24.603 & 8.945 & 36,4 & 17.047 & 7.821 & 45,9 \\
\hline
\end{tabular}




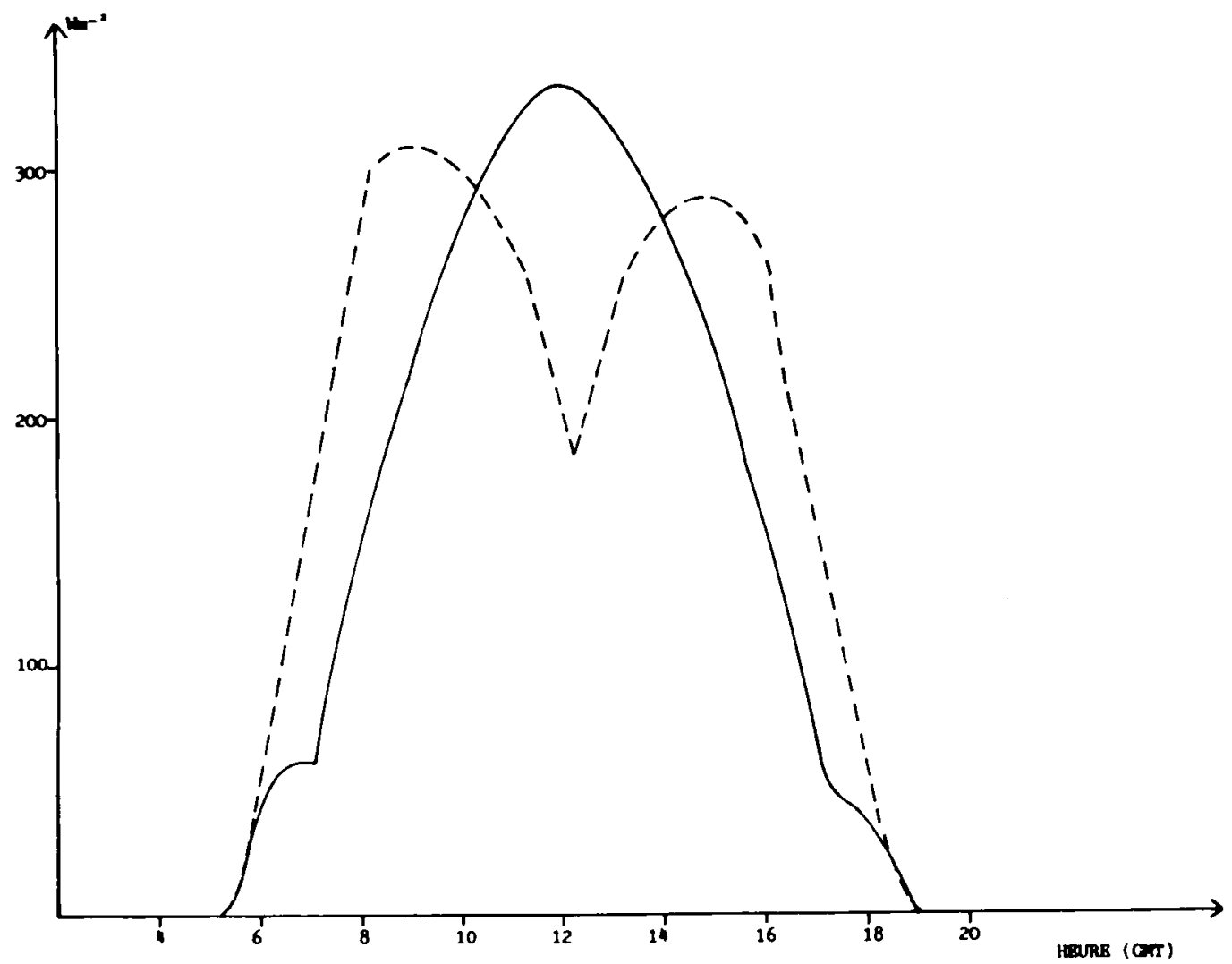

Fig. 7. 20 août.

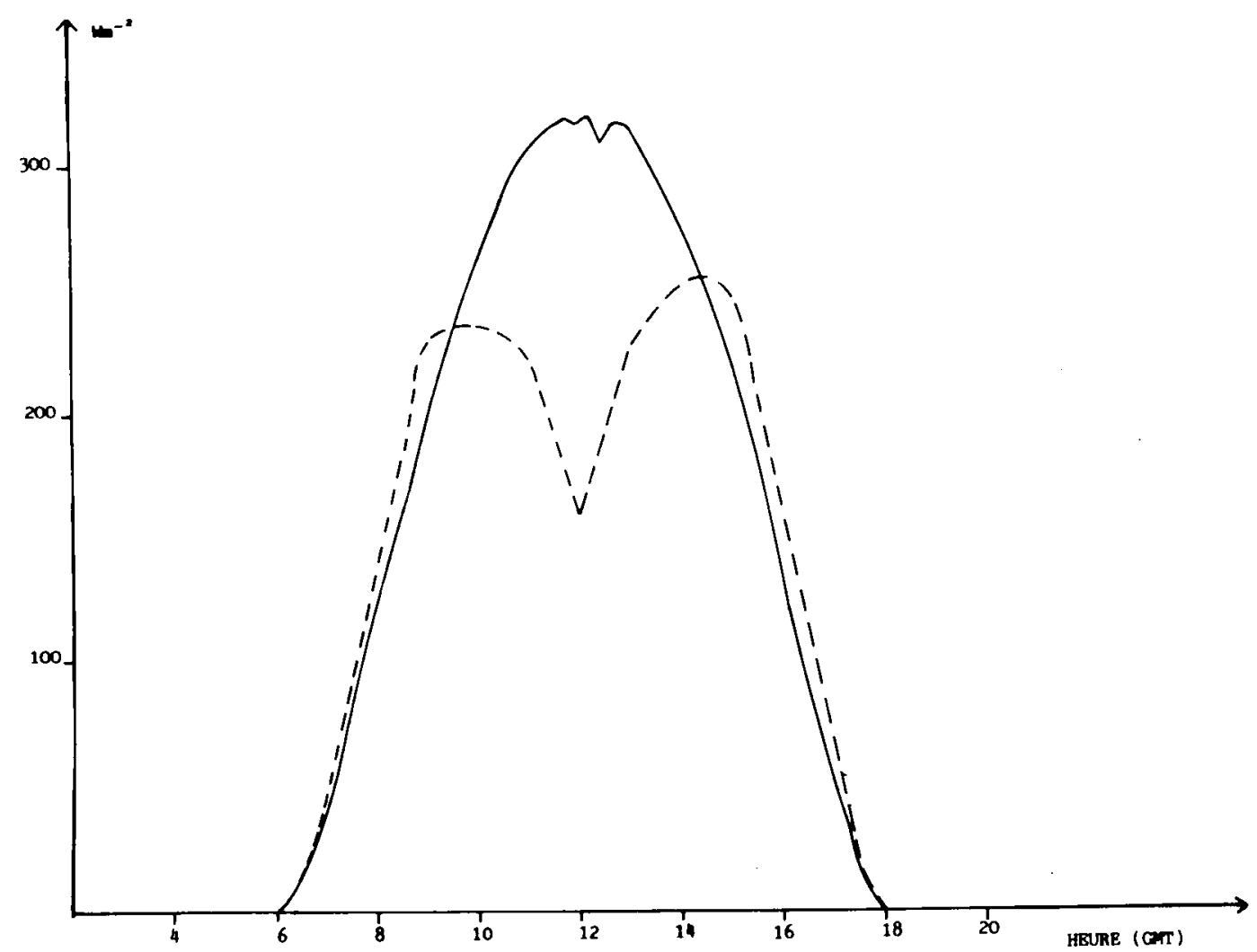

Fig 8. Equinoxe d'automne. 
Les coefficients d'interception sont peu variables pour l'orientation $\mathrm{N}-\mathrm{S}$, ils varient beaucoup plus pour les rangs $\mathrm{E}-\mathrm{O}$.

\section{Conclusion}

Le modèle géométrique simple adopté - un volume parallélépipédique creux aux faces horizontales pleines et aux faces verticales présentant une porosité constante quelle que soit la position du soleil - s'adapte bien à la vigne traditionnelle bordelaise qui possède une forme géométrique stricte et un indice foliaire réduit. Ce modèle est plus simple que la plupart de ceux qui sont décrits dans la littérature et permet d'évaluer l'absorption du rayonnement solaire direct aussi bien que celui du diffus, en tenant également compte de l'énergie rayonnante réfléchie par le sol.

La vérification expérimentale du modèle a montré un bon accord entre les mesures et les valeurs calculées du rayonnement solaire absorbé par la vigne.

Un tel modèle doit pouvoir convenir à d'autres cultures, quand l'occupation du sol par les plantes peut être représentée par des volumes parallélépipédiques, en rangs, et quand l'indice folaire est faible. L'introduction d'une valeur donnée de la porosité correspond mieux alors à la réalité que l'application d'une loi d'extinction (voir aussi Fuchs et Stanhill, 1980, pour le sorgho). Le modèle doit également convenir pour des rangs de végétation dense avec $p=1$.

Un tel modèle a permis de comparer l'absorption de l'énergie solaire à trois périodes importantes de la vigne, et pour deux orientations des rangs rencontrées dans la région. Sur l'ensemble des trois simulations, l'orientation Nord-Sud l'emporte sur l'orientation Ouest-Est.
Les éléments de calcul introduits ici pourront conduire à une évaluation du bilan radiatif total de la vigne avec prise en compte des échanges de grande longueur d'onde et fournir ainsi des informations intéressantes pour l'appréciation du bilan hydrique de la vigne.

\section{Références}

Allen L.H. (1974) Model of light penetration into a wide-row crop. Agron. J. 66, 41-47

Carbonneau A. \& Huglin P. (1980) Adaptation of training systems to French regions. Grape and Wine centennial symposium proceedings. Department of Viticulture and Enology. University of California, Davis, 376-385

Charles-Edwards D.A. \& Thorpe M.R. (1976) Interception of diffuse and direct-beam radiation by a hedgerow apple orchard. Ann. Bot. 40, 603-613

Fuchs M. \& Stanhill G. (1980) Row structure and foliage geometry as determinants of the interception of light rays in a sorghum row canopy. Plant Cell Environ. 3, 175-182

Jackson J.E. \& Palmer J.W. (1972) Interception of light by model hedgerow orchards in relation to latitude, time of year and hedgerow configuration and orientation. J. App. Ecol. 9, 341-357

Mann J.E., Curry G.L., Demichele D.W. \& Baker D.N. (1980) Light penetration in a row-crop with random plant spacing. Agron. J. 72, 131-139

Norman J.M. \& Welles J.M. (1983) Radiative transfer in an array of canopies. Agron. J. 75, 481-488

Riou C., Pieri P. \& Valancogne C. (1987) Variation de la vitesse du vent à l'intérieur et au-dessus d'une vigne. Agric. For. Météorol. 39, 143-154

Sellers W.D. (1965) Physical Climatology. The University of Chicago Press, Chicago, pp. 23-25

Smart R.E. (1973) Sunlight interception by vineyards. Amer. J. Enol. Viticul. 24, 141-147

Varlet-Grancher C. (1975) Variation et estimation de l'énergie solaire reçue sur des plans d'inclinaison et d'azimut variables. Ann. Agron. 26, 245-264 